\title{
Expression of miRNAs-122, -192 and -499 in end stage renal disease associated with acute myocardial infarction
}

\author{
Lobna Abdelsalam ${ }^{1}$, Alshaymaa A. Ibrahim², Aliaa Shalaby², Noha Osman³, Assem Hashad4, \\ Dina Badawy ${ }^{5}$, Hany Elghobary ${ }^{1}$, Eman Amer ${ }^{6}$
}

\author{
${ }^{1}$ Clinical and Chemical Pathology Department, Faculty of Medicine, Cairo University, \\ Cairo, Egypt \\ ${ }^{2}$ Clinical and Chemical Pathology Department, National Research Centre, Cairo, Egypt \\ ${ }^{3}$ Nephrology Department, Faculty of Medicine, Cairo University, Cairo, Egypt \\ ${ }^{4}$ Cardiology Department, Faculty of Medicine, Cairo University, Cairo, Egypt \\ ${ }^{5} \mathrm{Clinical}$ and Chemical Pathology department, Alzahraa University hospital, Al-azhar \\ University, Cairo, Egypt \\ ${ }^{6}$ Biochemistry Department, Faculty of Pharmacy, Ahram Canadian University, Cairo, \\ Egypt
}

Submitted: 6 March 2017

Accepted: 16 May 2017

Arch Med Sci 2019; 15 (5): 1247-1253

DOI: https://doi.org/10.5114/aoms.2019.87095

Copyright $\odot 2019$ Termedia \& Banach

\section{Abstract}

Introduction: New diagnostic tools are needed to accurately detect acute myocardial infarction (AMI) in patients with end stage renal disease (ESRD) presenting with ischemic chest pain. We aimed in this study to investigate circulating miR-122, -192 and -499 expression levels in patients with AMI on top of ESRD and evaluate the potential of these miRNAs as blood-based biomarkers for AMI in patients with ESRD.

Material and methods: The study included 80 ESRD patients without AMI, 80 patients with ESRD associated with AMI and 60 healthy subjects. Assessment of microRNAs was done using SYBR Green based real-time PCR.

Results: Levels of miR-122 were 28-fold and 20-fold higher in controls than in ESRD patients with or without AMI respectively $(p<0.001)$, while no differences were detected between the two patient groups $(p=0.9)$. Levels of miR-192 showed a marked increase in ESRD patients with and without AMI compared to the control group (>500-fold, $>8000$-fold respectively, $p \leq 0.001)$. Patients who developed AMI had lower expression than ESRD patients without AMI $(p<0.001)$. Non-significant miR-499 elevation was found in ESRD patients without cardiac disease compared to the control group, while highly significant elevation of miR- 499 was demonstrated in ESRD patients who developed AMI compared to other ESRD patients and the control group (>100-fold, > 350-fold respectively, $p=0.001$ ).

Conclusions: Altered expression of miR-122 and -192 may contribute in pathogenesis of ESRD. MiR-192 and -499 may serve as potential biomarkers for AMI in ESRD. Further studies are needed to correlate these miRNAs with disease progression and outcome.

Key words: acute myocardial infarction, chronic kidney disease, end stage renal disease, miRNA.

\section{Introduction}

Chronic kidney disease (CKD) is a growing health problem with prevalence of $10-13 \%$ around the world. It is classified into five stages based
Corresponding author: Alshaymaa A. Ibrahim Clinical and Chemical Pathology Department National Research

Centre

El Buhouth St

Dokki, Cairo, Egypt Phone: +20 1006193988 Fax: +20 233370931, E mail: shaymaa_@@hotmail. com 
on presence of signs of kidney damage and the glomerular filtration rate [1]. Acute myocardial infarction (AMI) is a life-threatening disease that is characterized by sudden death of cardiac muscles, resulting in biochemical changes, occurring in vessels and blood [2,3]. AMI has a high prevalence in patients with CKD and end stage renal disease (ESRD) compared to healthy individuals. It is one of the leading causes of death in these patients [4].

Diagnosis of $\mathrm{AMI}$ between CKD patients on maintenance hemodialysis is challenging. Although ischemic chest pain is considered one of the major characteristics for the diagnosis of AMI, it loses its credibility in patients with CKD because it may be absent secondary to uremic neuropathy and there might be pain secondary to non-ischemic causes such as uremic pericarditis [4, 5]. However, diagnosis usually depends on analysis of laboratory biomarkers such as troponins but unfortunately troponins were found to be unsuitable for the diagnosis of AMI in CKD patients as they did not even coincide with the angiographic findings in uremic patients $[5,6]$. Thus, considerable attention has been directed recently to the mechanisms and factors implied in AMI development in these patients but limited data exist regarding miRNAs' involvement in the setting of AMI in patients with CKD.

MicroRNAs (miRNAs) are small ( 22 -nucleotide long), stable and non-coding RNAs that play a key role in post-transcriptional gene regulation [2]. They have been considered recently as potential diagnostic markers due to their relative stability in circulation and their representation of the original cell [7].

Several studies have demonstrated miRNAs' profiles related to specific organs, including the heart and kidney. Some studies indicate that miR192 is one of the renal-specific microRNAs [8] and others support the role of miRNAs, especially miR-122 and -499, in the pathogenesis and progression of cardiac diseases [2, 7]. However, the available data discussing expression of circulating miR-122,-192 and -499 in patients with AMI on top of CKD and ESRD are still very limited.

We aimed in this study to investigate expression of circulating miR-122,-192 and -499 levels in patients with AMI on top of ESRD and evaluate the potential of these miRNAs as blood-based biomarkers for $\mathrm{AMI}$ in patients with ESRD.

\section{Material and methods}

\section{Subjects}

This case control study included 220 subjects recruited from different centers. Subjects were categorized into three groups.
Group 1 included 80 ESRD patients without AMI, recruited from the Nephrology Department of El-Kasr El-ainy Hospital. All patients satisfied the following criteria of ESRD stage 5 (G5): (1) the presence of kidney damage or decreased kidney function for 3 or more months, irrespective of the cause; (2) glomerular filtration rate (GFR) $15 \mathrm{ml} / \mathrm{min}$ per $1.73 \mathrm{~m}^{2}$ and treatment by dialysis [9]. All patients showed normal electrocardiograms and no current or previous history of cardiovascular diseases (CVDs).

Group 2 included 80 ESRD (G5) patients (described above) with AMI admitted to the emergency department of El-Kasr El-ainy hospital. All patients fulfilled the following inclusion criteria: (1) Admission was within the first $12 \mathrm{~h}$ of onset of ischemic-type chest pain lasting for longer than $30 \mathrm{~min}$; (2) presence of electrocardiographic ST segment elevation or presence of elevated serum CK-MB. Patients who had previous history of cardiac problems or were admitted more than $12 \mathrm{~h}$ after the onset of chest pain or had any hematological disease, acute or chronic infection, significant hepatic dysfunction, or any malignancies, were excluded from the study.

Group 3 included 60 age- and sex-matched healthy subjects who served as a control group. They were recruited from the National Research Centre while seeking a routine health check-up. These healthy subjects met the following criteria: (1) no evidence of any kidney or cardiac disease; (2) no evidence of associated systemic or chronic diseases or malignancy.

The protocol for this study was approved by Cairo University Ethics Committee and was according to the ethical guidelines of the Declaration of Helsinki with informed consent taken from all participated individuals.

\section{Sample collection and storage}

Blood specimens $(5 \mathrm{ml})$ were collected immediately after admission and within $12 \mathrm{~h}$ after the symptom onset in the AMI group. All blood samples were collected and divided into 2 EDTA-containing tubes. Thus, the $1^{\text {st }}$ tube was centrifuged at $4^{\circ} \mathrm{C}$ at $3,000 \mathrm{rpm}$ for $10 \mathrm{~min}$ to separate plasma. The supernatants were obtained then CK-MB mass was measured using Elecsys (Roche Diagnostics) and the $2^{\text {nd }}$ tubes were stored in a $-80^{\circ} \mathrm{C}$ freezer for further RNA analysis.

\section{Micro-RNA analysis using reverse transcriptase quantitative PCR (qRT-PCR) technique}

miRNAs were extracted using RNeasy Mini Kit (QIAGEN, Hilden, Germany), then the extracted miRNAs were reverse transcribed using a miScript reverse transcription (RT) kit (Qiagen, Hilden, Ger- 
many) then specimens of cDNA were stored at $-40^{\circ} \mathrm{C}$. Expression level of miRNAs was measured using SYBR Green based real-time PCR on a LightCycler 2.0 instrument (Roche Applied Science, Germany), according to the manufacturer's instructions. The relative levels of expression of miRNAs were computed using the comparative computed tomography (CT) method. MiRNA expression was normalized to GAPDH as a housekeeping gene

\section{Statistical analysis}

Analysis was performed using SPSS version 17 software for Windows. Data are reported as means \pm standard deviation (SD) or number (percentage). One-way ANOVA analysis was used for multiple group comparisons. The least significant difference (LSD) $t$ test was used in pairwise comparison of averages among groups. Linear regression analysis was done to confirm association of significant miRNA expression with the disease after adjustment of associated covariates. Receiver operating characteristic (ROC) analysis was used to assess the biomarker potential for the disease, and areas under the curves (AUCs) were reported. All tests were two-sided, and $p<0.05$ was considered statistically significant.

\section{Results}

Table I shows demographic and clinical data of studied groups.

Levels of miR-122 were 28-fold and 20-fold higher in control subjects than in ESRD patients with or without AMI $(p<0.001)$, while no differences were detected between the two patient groups $(p=0.9)$ (Figure $1 \mathrm{~A}$ ).

Serum levels of miR-192 showed a marked increase in ESRD patients with and without AMI compared to the control group (> 500-fold, $>$ 8000-fold respectively, $p \leq 0.001$ ). However, ESRD patients who developed AMI had lower expression than ESRD patients without AMI $(p<0.001)$ (Figure 1 B).

Slight non-significant miR-499 elevation was found in renal patients without cardiac disease compared to the control group, while highly significant elevation of miR-499 was demonstrated

Table I. Demographic and clinical data of the studied groups

\begin{tabular}{|c|c|c|c|c|}
\hline Parameter & $\begin{array}{l}\text { Control } \\
(n=60)\end{array}$ & $\begin{array}{l}\text { ESRD without AMI } \\
\quad(n=80)\end{array}$ & $\begin{array}{l}\text { ESRD with AMI } \\
\quad(n=80)\end{array}$ & $P$-value \\
\hline Age [years] & $56.8 \pm 6.3$ & $56.2 \pm 2.9$ & $56.6 \pm 5.8$ & 0.8 \\
\hline \multicolumn{4}{|l|}{ Sex: } & \multirow[t]{3}{*}{0.5} \\
\hline Female & $27(45.0 \%)$ & $29(36.0 \%)$ & $34(42.0 \%)$ & \\
\hline Male & $33(55.0 \%)$ & $51(64.0 \%)$ & $46(58.0 \%)$ & \\
\hline $\mathrm{BMI}\left[\mathrm{kg} / \mathrm{m}^{2}\right]$ & $27 \pm 3.5^{*}$ & $23.5 \pm 4.4$ & $23 \pm 4.8$ & 0.001 \\
\hline \multicolumn{4}{|c|}{ Family history of cardiac disease: } & \multirow[t]{3}{*}{0.3} \\
\hline Yes & $20(33 \%)$ & $31(39.0 \%)$ & $36(45.0 \%)$ & \\
\hline No & $40(67 \%)$ & $49(61 \%)$ & $44(55.0 \%)$ & \\
\hline \multicolumn{4}{|l|}{ Hypertension: } & \multirow[t]{3}{*}{0.001} \\
\hline Yes & $0(0 \%)$ & $38(48 \%)$ & $46(58 \%)$ & \\
\hline No & $60(100 \%)$ & $42(52 \%)$ & $34(42 \%)$ & \\
\hline \multicolumn{4}{|l|}{ Diabetes: } & \multirow[t]{3}{*}{0.001} \\
\hline Yes & $0(0 \%)^{*}$ & $50(62 \%)$ & $55(69 \%)$ & \\
\hline No & $60(100 \%)$ & $30(38 \%)$ & $25(31 \%)$ & \\
\hline \multicolumn{4}{|l|}{ Smoking: } & \multirow[t]{3}{*}{0.8} \\
\hline Yes & $25(42 \%)$ & $30(37 \%)$ & $33(41 \%)$ & \\
\hline No & $35(58 \%)$ & $50(63 \%)$ & 47 (59\%) & \\
\hline Urea [mg/dl] & $28.5 \pm 6.9^{*}$ & $126.6 \pm 17.5$ & $124.7 \pm 15.2$ & 0.001 \\
\hline Creatinine [mg/dl] & $0.7 \pm 0.2^{*}$ & $10.3 \pm 1.6$ & $10 \pm 1.4$ & 0.001 \\
\hline
\end{tabular}

Results presented as mean $\pm S D$ or frequency (percentage). ESRD - end stage renal disease, AMI-acute myocardial infarction, BMI-body mass index. *Significant $p$-value between patients and controls. 
A

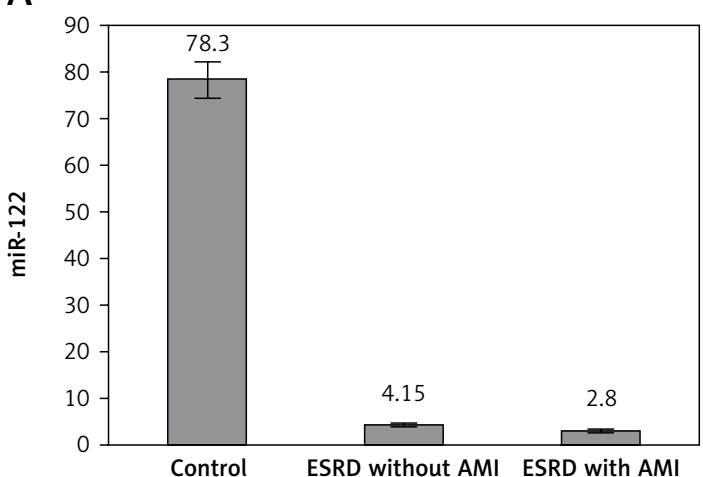

C

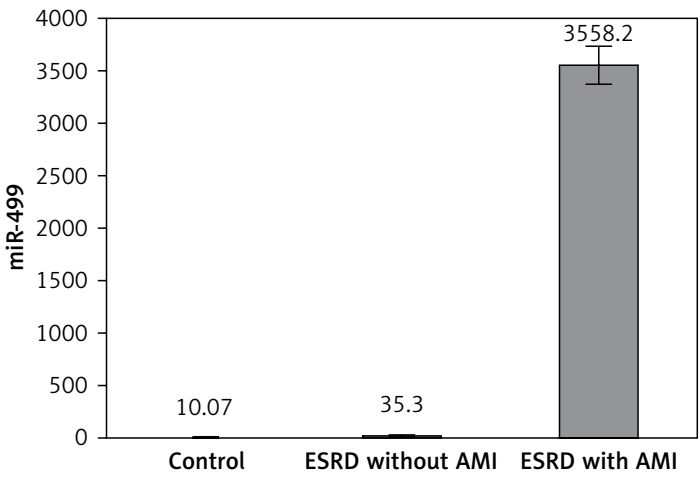

in ESRD patients who developed AMI compared to other ESRD patients and the control group ( $>100$ fold, > 350-fold respectively, $p=0.001$ ) (Figure 1 C). Regression analysis confirmed the association of altered levels of miR-499 with risk of AMI development in ESRD patients even after adjustment for age, sex, BMI, family history of cardiac disease, smoking, hypertension and diabetes $(p=0.001)$. Regression analysis also revealed no association of miR-499 with other risk factors including family history of cardiac disease, smoking, hypertension and diabetes ( $p=0.6, p=0.4, p=0.1, p=0.4$ ).

ROC analysis was performed to evaluate the usefulness of miR-122, -192 and -499 as a potential blood-based biomarker for AMI in ESRD and revealed AUC of $62 \%, 99.5 \%$ and $100 \%$ respectively, suggesting the role of miR-192 and -499 in discriminating ESRD patients with AMI from those without (Table II, Figure 2).
B

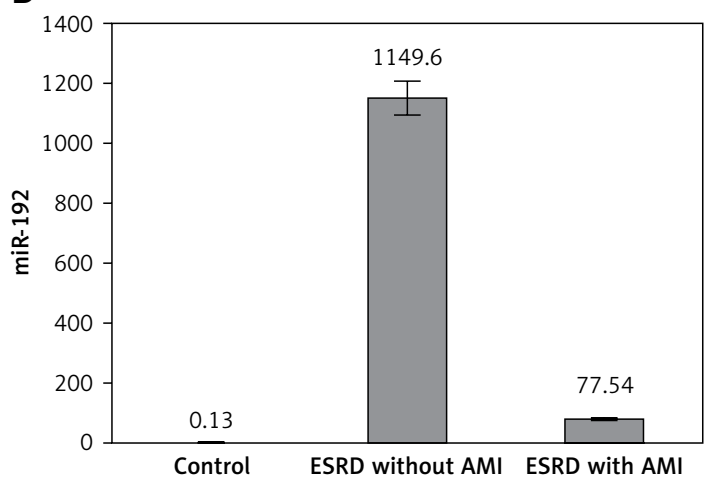

Figure 1. Mean expression of miR-122 (A), miR-192

(B) and miR-499 (C) in the studied groups

ESRD - end stage renal disease, AMI - acute myocardial infarction.

\section{Discussion}

AMI is one of the leading causes of mortality in patients with CKD. The diagnosis of AMI mainly depends on clinical presentation and ECG changes [6]. However, both clinical presentation and ECG changes are non-specific especially in early AMI. At present, circulating cardiac biomarkers such as troponins and CK-MB are more important for the diagnosis of AMI, but they have slow-release patterns and lack appropriate sensitivity and specificity in early AMI $[4,5]$. Therefore, it is necessary to seek new sensitive and specific biomarkers to accurately detect AMI in patients with CKD.

Several studies have described miR-122 as one of the liver-specific miRNAs, playing a role in the regulation of lipid and cholesterol metabolism [7]. However, reports regarding miR-122 in CKD are very few. In this study, levels of miR-122 were 28 -fold and 20 -fold higher in control subjects

Table II. Diagnostic performance of studied miRNAs for discriminating ESRD patients with AMI from those without AMI

\begin{tabular}{|lccccccc|}
\hline Marker & $\begin{array}{c}\text { Marker } \\
\text { cutoff }\end{array}$ & AUC & Sensitivity & Specificity & $\begin{array}{c}\text { Positive } \\
\text { predictive } \\
\text { value (PPV) }\end{array}$ & $\begin{array}{c}\text { Negative } \\
\text { predictive } \\
\text { value (NPV) }\end{array}$ & $\begin{array}{c}\text { Diagnostic } \\
\text { accuracy }\end{array}$ \\
\hline miR-122 & 0.027 & $62.0 \%$ & 88.8 & 50.0 & 64.0 & 81.6 & 69.4 \\
\hline miR-192 & 402.0 & $99.5 \%$ & 93.8 & 100.0 & 100.0 & 94.1 & 96.9 \\
\hline miR-499 & 53.160 & $100.0 \%$ & 100.0 & 100.0 & 100.0 & 100.0 & 100.0 \\
\hline
\end{tabular}

miRNA = miR - micro RNA, ESRD - end stage renal disease, AMI - acute myocardial infarction, AUC - area under the curve. 
than in ESRD patients with and without AMI. An experimental microarray analysis study revealed decreased expression of miR-122 in acute kidney injury [10]. Decreased miR-122 expression in renal disease could be explained by the previous mentioned findings of a link between downregulation of miR-122 and transforming growth factor- $\beta$ (TGF- $\beta$ ), which is considered as a potent cytokine that stimulates fibrinogenesis and consequently results in accumulation of fibroblasts and matrix proteins leading to progressive kidney disease $[11,12]$. In the current study, no differences were detected in miR-122 levels between ESRD patients who developed AMI and ESRD patients without AMI. Therefore, it will not be an optimal marker of the presence or absence of myocardial injury in patients with CKD. However, its clinical relevance in the diagnosis of $\mathrm{AMI}$ remains under research and published data regarding miR-122 in cardiac diseases are controversial. D'Alessandra et al. reported that miR-122 was expressed at very low levels in cardiac muscles, but they also found no significant differences in plasma levels of miR-122 in mouse models and AMI patients [13]. Our results disagree with other studies that reported upregulation of miR-122 in acute coronary syndromes (ACS) [14-16]. D'Alessandra et al. mentioned that the ACS group in their study had higher levels of miR-122 than the non-ACS (chronic heart disease + stable angina) group [14]. Li et al. found that levels of miR-122 were elevated earlier than troponin, suggesting their efficiency in early diagnosis [15]. Chen et al. also found significant elevation of serum miR-122 levels in AMI patients compared to the control group [16]. Published data regarding miR-122 in AMI are inconsistent but may be partially explained by the different characteristics of animals and patients, sample size and type, and time of sample withdrawal after onset of the infarction.

miR-192 is highly expressed in the healthy kidney, compared to other organs [17]. Altered level of miR-192 has been associated with multiple kidney diseases. In the current study, we found higher levels of serum miR-192 in ESRD patients with or without AMI compared to healthy controls. Our results agree with experimental and human studies demonstrating increased expression of miR-192 in diabetic nephropathy [17-19]. Wang et al. demonstrated that miR-192 was upregulated in human renal tissues of hypertensive glomerulosclerosis [20]. Cai et al. reported higher levels of serum miR192 in primary focal segmental glomerulosclerosis compared to patients with minimal change disease and healthy controls [21]. Our results disagree with a recent study reporting that down-regulation of miR-192 in renal tissue promotes fibrogenesis in patients with diabetic nephropathy [22].

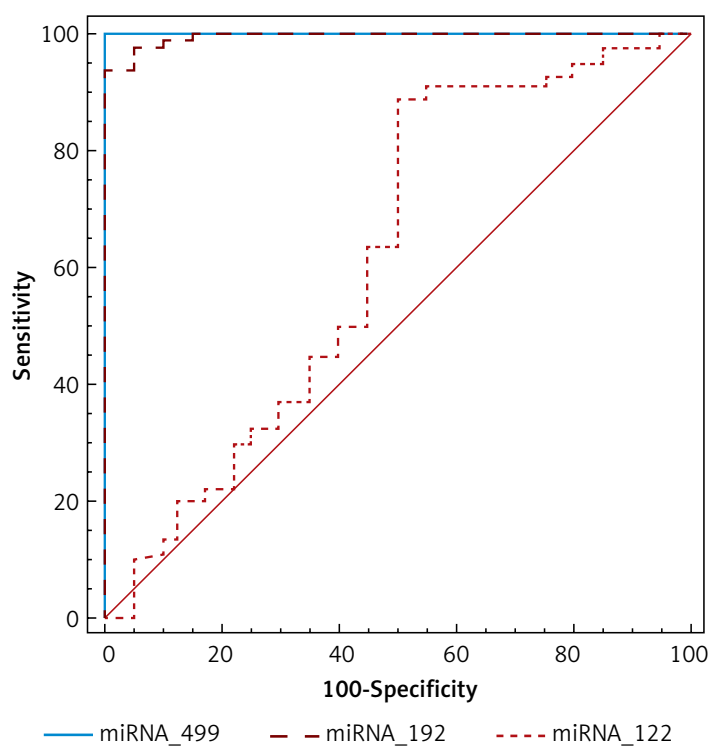

Figure 2. Receiver operating characteristic (ROC) curves of the studied miRNAs in differentiation of end stage renal disease with acute myocardial infarction from those without

Szeto et al. demonstrated lower urinary expression of miR-192 in patients with active lupus nephritis than healthy controls [23]. Wang et al. also reported that loss of miR-192 is associated with renal fibrosis [24]. Downregulation of miR-192 was also reported in models of experimental diabetic nephropathy [25]. The different findings reported in expression of miR-192 in human and animal models in kidney diseases might result from the different etiology and pathology of CKD populations included in the study, or differences in sample type and size. We also reported that ESRD patients who developed AMI had lower miR-192 expression than ESRD patients without AMI. This finding may be explained by different published data describing altered expression of TGF- $\beta 1$ and p53 in AMI patients, leading to downregulation of miR-192 [26-29]. However, Matsumoto et al. found an increased serum level of miR-192 in AMI patients after 18 days of onset of infarction and this level correlated with onset of heart failure within one year after the infarction. The contrasting findings mentioned above shed light on the complex nature of miRNA research. Some of the differences may relate to the complex regulatory networks that affect expression of miRNAs where their actions are coordinated, complementary and affected by different epigenetic and proteomic factors related to disease conditions [30].

In the current study, miR-499 showed slight non-significant elevation in patients without AMI compared to controls and showed significantly higher expression in $\mathrm{AMI}$ patients than controls and other ESRD patients. These results partially disagree with Emilian et al., who found that 
expression of miR-499 was > 80-fold higher in patients with ESRD than controls but they also mentioned that miR-499 decreased dramatically under the effect of dialysis and this last reported result could also explain the non-significant elevation in our study because all patients were under dialysis [31]. However, our results agree with consistent published data describing miR-499 as one of the exclusive cardiac miRNAs [2, 32]. Shalaby et al. found that miR-499 was significantly increased in patients with ACS compared with non-cardiac chest pain patients and ROC curve analysis revealed AUC $=0.98$ for the diagnosis of ACS [32]. A meta-analysis of eight studies covering 1634 participants which evaluated the diagnostic value of miR-499 in AMI revealed satisfactory sensitivity and specificity values of miR-499 (88\%, 87\% respectively) [33]. Zhang et al. reported that miR-499 was highly elevated in $\mathrm{AMI}$ patients compared with the non-AMI group and healthy subjects and the AUC of miR-499 for the diagnosis of AMI was 0.86 [34]. In this study, miR-499 showed the highest diagnostic accuracy between the studied miRNAs, suggested its potential role in diagnosis of early AMI in ESRD patients. However, high sensitivity and specificity $(100 \%, 100 \%$ respectively) reported in this study in comparison to previous studies could be partially explained by the small sample size and huge differences in expression levels between studied groups.

In conclusion, altered expression of miR-122 and miR-192 may contribute to the pathogenesis of CKD and ESRD and miR-192 and -499 may serve as potential biomarkers for AMI in ESRD. Further studies are needed to correlate levels of these miRNAs with disease progression and outcome.

\section{Acknowledgments}

This study was performed in the National Research Centre and Cairo University.

\section{Conflict of interest}

The authors declare no conflict of interest.

\section{References}

1. Colombo G, Reggiani F, Cucchiari D, et al. Plasma protein-bound di-tyrosines as biomarkers of oxidative stress in end stage renal disease patients on maintenance haemodialysis. BBA Clin 2017; 7: 55-63.

2. Shalaby A, Ibrahim A, Elghobary H, Youssef G. Detection of micro RNA-499 in acute myocardial infarction, significance of a new marker. RJPBCS 2017; 8: 1480-5.

3. Piwowar A, Knapik-Kordecka M, Warwas M. Ischemiamodified albumin level in type 2 diabetes mellitus - preliminary report. Dis Markers 2008; 24: 311-7.

4. Jafari Fesharaki M, Alipour Parsa S, Nafar M, GhaffariRahbar M, Omidi F, Karimi-Sari H. Serum troponin I level for diagnosis of acute coronary syndrome in patients with chronic kidney disease. Iranian Jf Kidney Dis 2016; 10: 11-6.

5. Franczyk-Skóra B, Gluba A, Banach M, Rysz J. Treatment of non-ST-elevation myocardial infarction and ST-elevation myocardial infarction in patients with chronic kidney disease. Arch Med Sci 2013; 9: 1019-27.

6. Ross BA, Wald R, Goldstein MB, et al. Relationships between left ventricular structure and function according to cardiac MRI and cardiac biomarkers in end-stage renal disease. Can J Cardiol 2017; 33: 501-7.

7. Cortez-Dias N, Costa MC, Carrilho-Ferreira P, et al. Circulating miR-122-5p/miR-133b ratio is a specific early prognostic biomarker in acute myocardial infarction. Circ J 2016; 80: 2183-91.

8. Virzì GM, Clementi A, Brocca A, de Cal M, Ronco C. Molecular and genetic mechanisms involved in the pathogenesis of cardiorenal cross talk. Pathobiology 2016; 83: 201-10.

9. National Kidney Foundation K/DOQI clinical practice guidelines for chronic kidney disease: evaluation, classification and stratification. Am J Kidney Dis 2002; 39: S1-266.

10. Lee CG, Kim JG, Kim HJ, et al. Discovery of an integrative network of microRNAs and transcriptomics changes for acute kidney injury. Kidney Int 2014; 86: 943-53.

11. Zeng C, Wang YL, Xie C, et al. Identification of a novel TGF-beta-miR-122-fibronectin 1/serum response factor signaling cascade and its implication in hepatic fibrogenesis. Oncotarget 2015; 6: 12224-33.

12. Lan HY. Diverse roles of TGF-beta/Smads in renal fibrosis and inflammation. Int J Biol Sci 2011; 7: 1056-67.

13. D’Alessandra Y, Devanna P, Limana F, et al. Circulating microRNAs are new and sensitive biomarkers of myocardial infarction. Eur Heart J 2010; 31: 2765-73.

14. D’Alessandra Y, Carena MC, Spazzafumo L, et al. Diagnostic potential of plasmatic microRNA signatures in stable and unstable angina diagnostic potential of plasmatic microRNA signatures in stable and unstable angina. PLoS One 2013; 8: e80345.

15. Li X, Yang Y, Wang L, et al. Plasma miR-122 and miR3149 potentially novel biomarkers for acute coronary syndrome. PLoS One 2015; 10: e0125430.

16. Chen P, Yu M, Yan L, Wu Y. Diagnostic value of serum miR-122 in acute myocardial infarction. Int J Clin Exp Pathol 2016; 9: 1788-93.

17. Chung AC, Yu X, Lan HY. MicroRNA and nephropathy: emerging concepts. Int J Nephrol Renovasc Dis 2013; 6: 169-79.

18. Trionfini P, Benigni A, Remuzzi G. MicroRNAs in kidney physiology and disease. Nature Rev Nephrol 2015; 11 : 23-33.

19. Chien HY, Chen CY, Chiu YH, Lin YC, Li WC. Differential microRNA profiles predict diabetic nephropathy progression in Taiwan. Int J Med Sci 2016; 13: 457-65.

20. Wang G, Kwan BC, Lai FM, et al. Intrarenal expression of miRNAs in patients with hypertensive nephrosclerosis. Am J Hypertens 2010; 23: 78-84.

21. Cai X, Xia Z, Zhang C, et al. Serum microRNAs levels in primary focal segmental glomerulosclerosis. Pediatr Nephrol 2013; 28: 1797-801.

22. Krupa A, Jenkins R, Luo DD, Lewis A, Phillips A, Fraser D. Loss of microRNA-192 promotes fibrogenesis in diabetic nephropathy. J Am Soc Nephrol 2010; 21: 438-47.

23. Szeto CC, Ching-Ha KB, Ka-Bik L, et al. Micro-RNA expression in the urinary sediment of patients with chronic kidney diseases. Dis Markers 2012; 33: 137-44. 
24. Wang B, Herman-Edelstein M, Koh P, et al. E-cadherin expression is regulated by miR-192/215 by a mechanism that is independent of the profibrotic effects of transforming growth factor-beta. Diabetes 2010; 59: 1794-802..

25. Kasinath BS, Feliers S. The complex world of kidney microRNAs. Kidney Int 2011; 80: 334-7.

26. Kato M, Zhang J, Wang M, et al. MicroRNA-192 in diabetic kidney glomeruli and its function in TGF-beta-induced collagen expression via inhibition of E-box repressors. Proc Natl Acad Sci USA 2007; 104: 3432-7.

27. Euler G. Good and bad sides of TGFbeta-signaling in myocardial infarction. Front Physiol 2015; 6: 66.

28. Gogna R, Madan E, Khan M, Pati U, Kuppusamy P. p53's choice of myocardial death or survival: oxygen protects infarct myocardium by recruiting p53 on NOS3 promoter through regulation of p53-Lys 118 acetylation. EMBO Mol Med 2013; 5: 1662-83.

29. Matsumoto S, Sakata Y, Suna S, et al. Circulating p53responsive microRNAs are predictive indicators of heart failure after acute myocardial infarction. Circ Res 2013; 113: 322-6.

30. Kantharidis P, Wang B, Carew RM, Lan HY. Diabetes complications: the microRNA perspective. Diabetes 2011; 60: 1832-7.

31. Emilian C, Goretti E, Prospert F, et al. MicroRNAs in patients on chronic hemodialysis (MINOS study). Clin J Am Soc Nephrol 2012; 7: 619-23.

32. Shalaby SM, El-Shal AS, Shoukry A, Khedr MH, Abdelraheim N. Serum miRNA-499 and miRNA-210: a potential role in early diagnosis of acute coronary syndrome. IUBMB Life 2016; 68: 673-82.

33. Cheng C, Wang Q, You W, Chen M, Xia J. MiRNAs as biomarkers of myocardial infarction: a meta-analysis. PLoS One 2014; 9: e88566.

34. Zhang L, Chen X, Su T, et al. Circulating miR-499 are novel and sensitive biomarker of acute myocardial infarction. J Thorac Dis 2015; 7: 303-8. 